\title{
The Infall Component of the Modern Martian Carbon Cycle
}

\author{
MARC D. FrIES ${ }^{1}$ AND ANDREW STEELE ${ }^{2}$ \\ ${ }^{1}$ NASA Curation Office, Johnson Space Center. ${ }^{2}$ Carnegie \\ Earth and Planets, Washington, D.C. marc.d.fries@nasa.gov
}

The modern martian carbon cycle is reduced in flux and overall activity compared to the Earth, but is fundamentally similar in concept. Interchange is possible between a few sources: subsurface sources to include igneous-origin native martian carbon that has been observed in martian meteorites, infall from meteorites and cosmic dust, seasonal flux arising from polar ice, and interaction with the oxidized carbon atmosphere. This presentation will focus on the infall component of that cycle, especially where it pertains to carbon fluxes and sinks at the surface. Since landed missions interact directly with infall at the martian surface, understanding infall flux and composition is critical to interpretation of landed mission data.

The martian surficial carbon cycle draws upon several important fluxes. In the martian subsurface, prebiotic chemical processes produce light carbon species during the cooling of igneous bodies. This carbon features varying chemical composition produced as mineral species precipitate and the igneous body cools, producing depositional behavior reminiscent of the Bowen series for silicates. These species are exposed at the martian surface via volcanism (which may be absent today), aeolean erosion, and turnover via meteoritic impact. In the atmosphere, carbon is dominated by carbon oxide gases buffered by carbon dioxide ice caps. Trace-level methane occurs as both a minor background species and in poorly-understood episodic events. Infall, by contrast, contributes a dominant flux to the modern martian surface. While infall is a minor element in the Earth's carbon cycle, the lack of modern volcanism, biology, oceans, and crustal recycling on Mars leave meteoritic infall as the most volunimous carbon flux onto the martian surface. Of note is the fact that infall carbon does not accumulate due to oxidation, generating a flux of carbon oxides to the atmosphere as well as carbon-depleted residuum onto the surface.

Mars' lack of oceans, biology, and active crustal recycling result in a much simpler carbon cycle than that on Earth. This is incredibly important from a scientific standpoint because Mars' relative simplicity preserves ancient prebiotic carbon cycling processes that must have been present on the early Earth. Those processes have been overwritten by biology on Earth but are directly available for study on Mars, at least until Mars is permanently disrupted by introduced terrestrial biology. 